\title{
HIGH-GRADIENT NORMAL-CONDUCTING RF STRUCTURES FOR MUON COOLING CHANNELS*
}

\author{
J. Corlett ${ }^{\dagger}$, M. A. Green, N. Hartman, A. Ladran, D. Li, R. MacGill, R. Rimmer, LBNL, Berkeley, CA, USA \\ A. Moretti, T. Jurgens, N. Holtkamp, FNAL, Batavia, IL, USA \\ E. Black, IIT, IL, USA \\ D. Summers, M. Booke, University of Mississippi, Oxford, MS, USA
}

\begin{abstract}
We present a status report on the research and development of high-gradient normal-conducting RF structures for the ionization cooling of muons in a neutrino factory or muon collider. High-gradient RF structures are required in regions enclosed in strong focussing solenoidal magnets, precluding the application of superconducting RF technology [1]. We propose using linear accelerating structures, with individual cells electromagnetically isolated, to achieve the required gradients of over $15 \mathrm{MV} / \mathrm{m}$ at $201 \mathrm{MHz}$ and $30 \mathrm{MV} / \mathrm{m}$ at $805 \mathrm{MHz}$. Each cell will be powered independently, and cell length and drive phase adjusted to optimise shunt impedance of the assembled structure. This efficient design allows for relatively small field enhancement on the structure walls, and an accelerating field approximately 1.7 times greater than the peak surface field. The electromagnetic boundary of each cell may be provided by a thin Be sheet, or an assembly of thin-walled metal tubes. Use of thin, low-Z materials will allow passage of the muon beams without significant deterioration in beam quality due to scattering. R\&D in design and analysis of robust structures that will operate under large electric and magnetic fields and RF current heating are discussed, including the experimental program based in a high-power test laboratory developed for this purpose.
\end{abstract}

\section{INTRODUCTION}

One of the most demanding applications of conventional conducting RF in a muon collider or neutrino factory is in ionization cooling of the muon beam. In such schemes, muons lose momentum in a low$\mathrm{Z}$ scattering material, or absorber (current designs use a liquid hydrogen absorber), after which they regain longitudinal momentum in a linear $\mathrm{RF}$ accelerating structure. Sections of RF and absorber are placed inside an alternating solenoidal magnetic field, which focuses the muon beam. A high RF accelerating field is required to rapidly restore the longitudinal momentum to the beam, allowing closely-packed scattering and acceleration sections, and in a time dictated by the short-lived muons.

$\mathrm{RF}$ systems are used for the purpose of matching the muon beam into the longitudinal acceptance of the cooling channel, and to replenish the longitudinal momentum lost by scattering in the hydrogen absorber.

\footnotetext{
* Work supported by the U.S. Department of Energy under contract No.s DE-AC0376SF00098 (LBNL), DE-AC0276CH00016 (FNAL), DE-FG0291ER40622 (University of Misissippi). †jncorlett@lbl.gov
}

The large transverse emittance and energy spread of the muon beam require a large RF bucket and a large physical aperture to contain the beam. Upstream sections of the cooling channel are designed with 201.25 MHz structures, and downstream structures, where the emittance is reduced by the action of previous cooling sections, are designed for $805 \mathrm{MHz}$ RF systems. Bunching and matching cavities are also used at $402.5 \mathrm{MHz}$. Transverse apertures at the irises of the $201.25 \mathrm{MHz}$ sections are required to be approximately $40 \mathrm{~cm}$, and $16 \mathrm{~cm}$ for the $805 \mathrm{MHz}$ sections. This aperture accounts for more than half of the diameter of a typical cavity at these frequencies (determined by the radius for a cylindrical $\mathrm{TM}_{010}$ mode pillbox). Such large bores lead to low shunt impedance in conventionally conducting open-iris structures. To increase the shunt impedance, we propose cavities with beam-pipe apertures electromagnetically bounded by thin windows of beryllium, or assemblies of thin-walled tubes of low-Z material. In addition to studies of these novel structures, we have begun measurements of the properties of a more conventional $\pi$-mode open-cell structure under the required conditions of accelerating RF field and static solenoidal magnetic field.

Initial studies have been focused on the smaller 805 $\mathrm{MHz}$ structures, a frequency at which we have readily available RF power sources, and at which structures are relatively small and inexpensive. Current and near-term work will focus on $201.25 \mathrm{MHz}$ structures, more appropriate for the neutrino factory, as well as the first stage cooling section for a collider. The larger structures at lower frequency present challenges in fabrication, control of thermal effects, and integration into a cooling channel.

An experimental area dedicated to the high-power testing of $805 \mathrm{MHz}$ structures for the neutrino factory and muon collider collaboration has been built in Lab-G at Fermilab, and a $201.25 \mathrm{MHz}$ facility is also planned.

\section{805 MHZ STRUCTURES}

Accelerating structure designs at $805 \mathrm{MHz}$ have been developed for both a pillbox-like cavity with Be foil windows, and for a $\pi$-mode open cell structure. The pillbox-like structure has a higher shunt impedance, while the open cell structure will be used to test achievable gradient in a more conventional design.

\section{$2.1805 \mathrm{MHz}$ Be Window Cavity}

Thin Be Foils are mounted in Be-alloy rings such that the foil is under tension after cool-down from the brazing 
process. To test the feasibility of such assemblies in highfield RF structures, a low-power test cavity has been built [2], and a high-power test cavity is being fabricated [3].

Low-power tests have been performed to assess the mechanical behavior of windows formed from thin (125 $\mu \mathrm{m})$ foils of pre-stressed beryllium under thermal load [4]. In the low-power test cavity, windows have been heated by $\sim 400 \mathrm{~W}$ RF power, and radiantly heated by a halogen lamp. The measurements have allowed determination of the detuning effects caused by displacement of the foil due to thermal expansion. The foils remain flat until the thermal expansion exceeds the tensile pre-stress. The maximum allowable temperature gradient across foils produced so far, before displacement occurs, is approximately $35^{\circ} \mathrm{C}$.

Measurements have also been performed on Be windows in a cavity cooled to liquid nitrogen temperature, at which the electrical and thermal conductivities of $\mathrm{Be}$ increase significantly. In this case no detuning of the RF cavity due to expansion of the Be foil was measured for power dissipation of up to $400 \mathrm{~W}$ in the cavity.

Small (approximately $1 \mathrm{~cm}$ diameter), $125 \mu \mathrm{m}$ thick Be foils have been tested to high gradient in the A0 facility at FNAL, and have survived surface fields of $35 \mathrm{MVm}^{-1}$ at $1.3 \mathrm{GHz}$.

An $805 \mathrm{MHz}$ high-power test cavity has been designed to accept demountable $16 \mathrm{~cm}$ diameter Be foil windows for testing to high gradient in the Lab-G facility. Figure 1 shows the cavity during fabrication; the input power coupler, and the steel rings to which the Be windows will be mounted can be seen. Windows of different designs may be tested in this cavity, and we plan to experiment with foils of various thickness, variation of the thickness as a function of radius, different alloys, and antimultipactor coatings. In the Lab-G facility we will be able to test conditioning and survivability of the larger foils at high gradient, and under various magnetic field configurations as expected in the cooling channel.

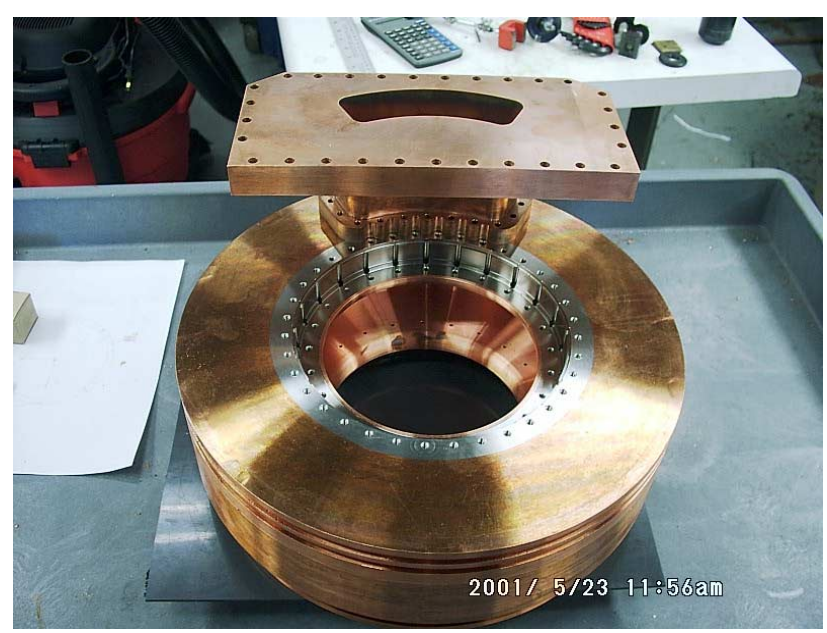

Figure 1.Be window high-power test cavity in fabrication.

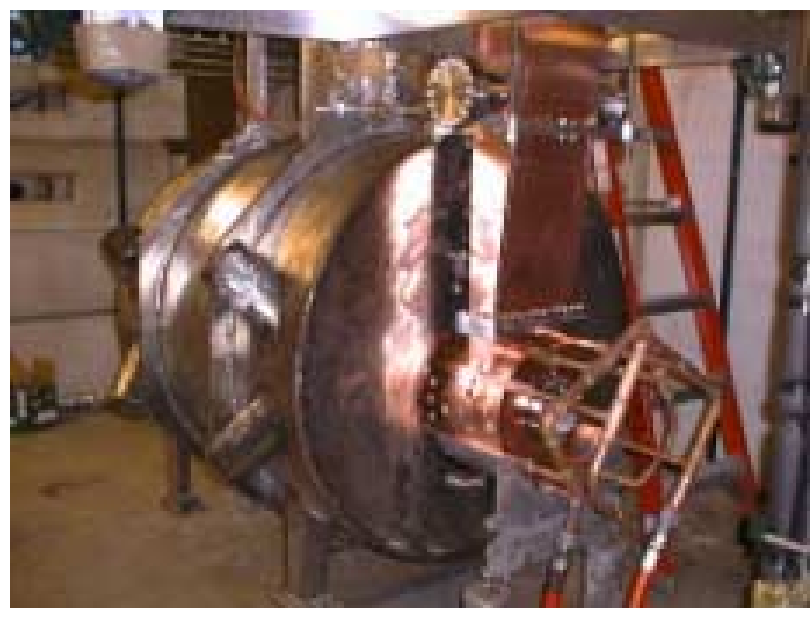

Figure 2. 6-cell open-cell structure installed in the superconducting magnet at the Lab G facility.

\section{$2.2805 \mathrm{MHz} \pi$-mode Open-Cell Cavity}

A 6-cell $\pi$-mode open-cell cavity designed to achieve the required gradient of $15 \mathrm{MVm}^{-1}$, with a shunt

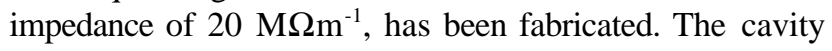
iris apertures are varied to follow the transverse beam profile of a cooling channel, and edges are rounded to reduce electric field enhancement. The calculated peak surface field is approximately $22 \mathrm{MVm}^{-1}$. Figure 2 shows the 6-cell $\pi$-mode open-cell cavity installed in the superconducting magnet in the Lab-G facility, where conditioning and achievable gradient will be tested

\section{LAB-G TEST FACILITY}

The Lab-G test facility at Fermilab has been developed for high-power testing of RF components at $805 \mathrm{MHz}$. The facility comprises a concrete bunker to shield x-rays during high-power operation of a cavity, a superconducting solenoidal magnet inside which the accelerating RF structures are placed, a high-power RF system with power fed into the bunker via waveguide, and cryogenics, water, controls, and interlocks systems. The facility is fully operational and the first cavity is under test.

\subsection{RF system}

An $805 \mathrm{MHz}$ klystron provides peak power of up to 10 MW in a 100 microsecond pulse, at up to $10 \mathrm{~Hz}$ repetition rate. Power is fed into the concrete shielded bunker through WR975 waveguide, with a ceramic vacuum window placed near the accelerating structure. Two vacuum pumps may be mounted on the UHV side of the window, and provide pumping for the cavity. Vacuum components are shielded from the magnetic field of the solenoid. A water circuit controllable to $\pm 0.1^{\circ} \mathrm{C}$ allows coarse control of cavity resonant frequency through water cooling channels wrapped around the cavity. The klystron bandwidth of $\pm 2 \mathrm{MHz}$ allows a feedback system to fine control the RF source frequency to follow the cavity resonance. 


\subsection{Superconducting solenoid}

The superconducting solenoid magnet contains a pair of coils, which may be independently powered to produce either a uniform field of up to 5T, or a gradient field at the center of the magnet. This system allows reproduction of the solenoidal fields in the $805 \mathrm{MHz}$ section of the cooling channel, and the study of multipacting effects as a function of solenoid field strength and gradient. Figure 3 shows results of field measurements in the magnet.

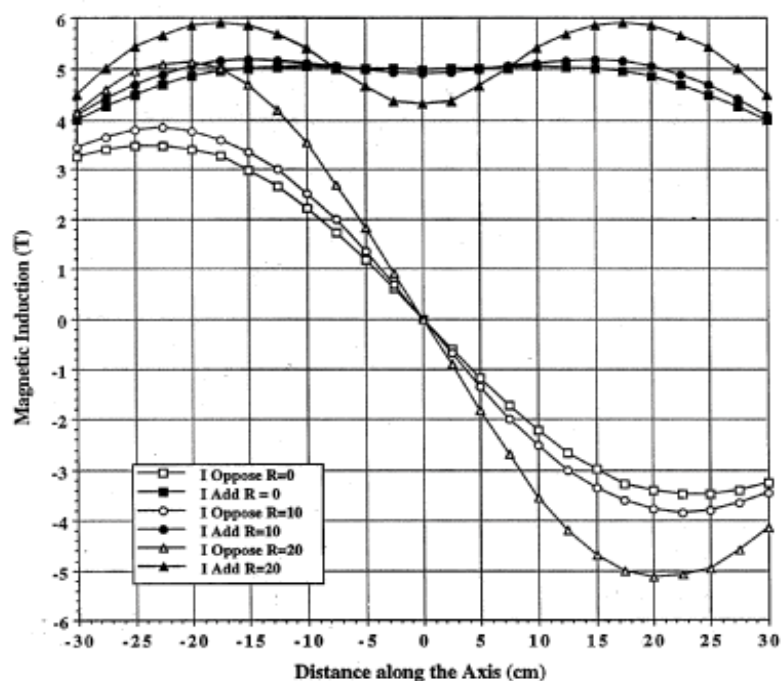

Figure 3. Superconducting solenoid field measurements.

\section{201 MHZ STRUCTURES}

The lower-frequency structures present additional challenges in fabrication and control of resonant frequency. In addition to improving the shunt impedance by use of flat-foil Be window boundaries to the cavities, we are also investigating other electromagnetic terminations formed by arrangements of thin-walled tubes fabricated from low$\mathrm{Z}$ metals, or double-walled foils. Such structures may have the advantage of increased mechanical strength and stability over the single flat foil, and may allow additional cooling by passing cold gas through the structures. A grid of thin-walled tubes produces transverse fields near their surfaces, but the resulting particle deflections are small compared to other scattering mechanisms.

Cavity cross-sections have been rounded to improve the quality factor. We expect a field enhancement as large as 1.7 on the aperture noses, however this is only 1.5 times the Kilpatrick limit of $15 \mathrm{MVm}^{-1}$ at this frequency [5]. The foils must be thick enough to conduct away heat generated by the RF currents, but not too thick to cause excessive scattering of the muon beam. For current designs, foil thickness varies from $100 \mu \mathrm{m}$ to $900 \mu \mathrm{m}$ on the beam axis, and thickness may also increase with radius for foils in the higher-gradient cavities.

Current designs are for "room temperature" operations, however the possibility of operating at reduced temperature (e.g. with liquid nitrogen cooling) to reduce wall resistance, window heating, and peak RF power requirements, remains under consideration.

\subsection{ANSYS ${ }^{\mathrm{TM}}$ modeling of Be windows}

An ANSYSTM model has been developed to analyze behavior of the thin-foil, pre-stressed windows. Predicted behavior under thermal loads expected from RF current heating is similar to that measured for the thin Be foils [6]. Figure 4 shows calculations of the displacement at the center of a $38 \mathrm{~cm}$ diameter pre-stressed foils of different thickness as a function of power dissipated in the foil. Similar calculations for a $16 \mathrm{~cm}$ foil show reasonable agreement with measurements.

ANSYS ${ }^{\mathrm{TM}}$ calculations including pre-stressed Be foils are in progress to determine optimal dimensions and fabrication techniques for the larger, lower-frequency sections of the cooling channel. Our designs will develop based on experience with Be foil windows in the $805 \mathrm{MHz}$ high-power test cavity at Lab-G.

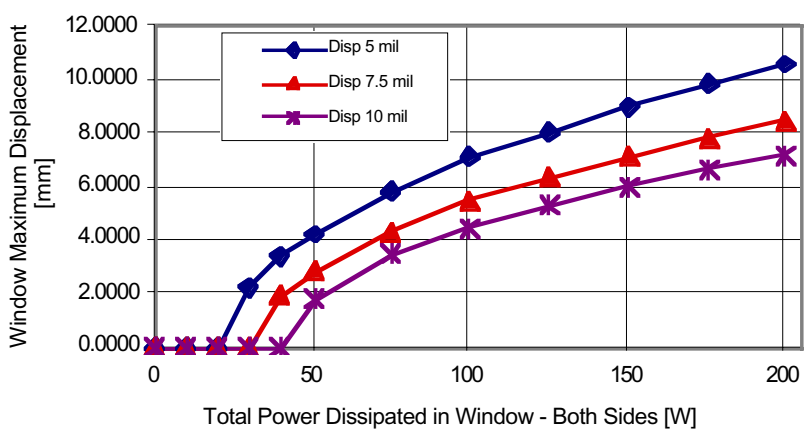

Figure 4. Calculated displacement of pre-stressed Be foil as a function of power dissipated in $38 \mathrm{~cm}$ diameter window thickness $127,190.5$, and $254 \mu \mathrm{m}$.

\section{REFERENCES}

[1] "RF Accelerating Structures for the Muon Cooling Experiment", J. Corlett, M. Green, H. Kirk, D. Li, A. Moretti, R. Palmer, D. Summers, Y. Zhao, N. Holtkamp, PAC'99, New York, March 29-April2, 1999.

[2] "Be window studies at room temperature", D. Li, J. N. Corlett, N. Hartman, S. Kar, M. Leung, D. Lozano, Mucool 110.

[3] "Design and Fabrication of an 805 MHz RF Cavity with Be windows for a High RF Power Testing for a Muon Cooling Experiment", Derun Li, J. Corlett, R. MacGill, R. Rimmer, D. Summers, M. Booke, this conference.

[4] "Deflection measurements of thin foils for the muon cooling RF channel", J.N. Corlett, N. Hartman, D. $\mathrm{Li}, \mathrm{D}$. Turler, Mucool 62

[5] "Closed-cell 201.25 MHz Structures for a Muon Cooling Channel", R. Rimmer, N. Hartman, A. Ladran, D. Li, Al. Moretti, T. Jurgens, this conference.

[6] "Thin Beryllium Windows - Analysis and Design Status", N. Hartman, D. Li, J. Corlett, Mucool 180 